\title{
UN NUEVO MESOEUCROCODYLIA CRETÁCICO DEL NORTE DE LA CUENCA NEUQUINA, ARGENTINA
}

\author{
ANDREA B. ARCUCCI \\ Universidad Nacional de San Luis, Chacabuco y Pedernera, 5700, San Luis, Argentina.arcucci@unsl.edu.ar \\ LEONARDO S. FILIPPI \\ Museo Municipal Argentino Urquiza, Rincón de los Sauces, Neuquén, Argentina.lsfilippi@gmail.com
}

JORGE O. CALVO

Centro Paleontológico Lago Barreales, Universidad Nacional del Comahue, Neuquén, Argentina. jorgecalvo@proyectodino.com.ar

\begin{abstract}
A NEW CRETACEOUS MESOEUCROCODYLIA FROM THE NORTH OF THE NEUQUÉN BASIN, ARGENTINA. In this contribution, a new specimen of Mesoeucrocodylia from levels attributed to the Plottier Formation (Cretaceous, Upper Coniacian) is described in the locality Yacimiento Narambuena, Rincón de los Sauces, in the north section of the Neuquén Province. The material includes an incomplete subadult specimen with mostly articulated postcranium of an approximate length of $60 \mathrm{~cm}$. The study and comparisons to allow distinguish the specimen from other Mesoeucrocodylia previously known from this same basin and suggest the presence of a new taxon, increasing the diversity of this clade in this period in the Neuquén Basin. The specimen from Rincon de los Sauces presents postcraneal characters previously attributed to Notosuchia and Neosuchia, both clades mainly defined by cranial characters. This set of primitive and derived characters prevents the location of its precise phylogenetic position among the Mesoeucrocodylia.
\end{abstract}

Key words: Cretaceous, Neuquén Basin, Coniaciano, Plottier Formation, Mesoeucrocodylia.

RESUMO - Um Mesoeucrocodylia é descrito para o afloramento Narambuena, em níveis da Formação Plottier (Coniaciano Superior), em Rincón de los Sauces (extremo norte da província de Neuquén). Trata-se de um espécime incompleto que inclui grande parte do pós-crânio articulado de um subadulto de comprimento total estimado em $60 \mathrm{~cm}$. O estudo e as comparações realizadas nos restos utilizados neste trabalho sugerem a presença de um táxon diferente aos reconhecidos previamente e incrementa a diversidade existente entre os Mesoeucrocodylia da bacia Neuquina. O exemplar de Rincón de los Sauces exibe caracteres do pós-crânio que tem sido originalmente atribuído a Notosuquia e Neosuchia, clados que têm sido diagnosticados principalmente por caracteres cranianos. Esse conjunto de caracteres primitivos e derivados impede a localização, no momento, de sua posição filogenética precisa entre os Mesoeucrocolylia.

Palavras-chave: Cretáceo, bacia Neuquina, Formação Plottier, Coniaciano, Mesoeucrocodylia.

\section{INTRODUCCIÓN}

Los Crocodyliformes tienen una larga historia evolutiva que comienza hacia el Triásico Superior, hace aproximadamente unos $220 \mathrm{Ma}$. Las especies actuales, de hábitos anfibios, constituyen una pequeña muestra dentro de la gran variedad de tipos adaptativos que se desarrollaron durante el Jurásico, Cretácico y gran parte del Terciario (Gasparini, 1981). En Argentina existen afloramientos mesozoicos que han brindado especímenes pertenecientes a los dos grandes linajes de Crocodyliformes: Protosuchia y Mesoeucrocodylia (incluyendo este último a Neosuchia, el grupo monofilético que incluye las formas actuales) con tipos adaptativos que incluyen desde animales terrestres (Notosuchia) a los exclusivamente marinos (Thalattosuchia) (Gasparini, 1981). Los Crocodyliformes del Cretácico Superior de la Cuenca
Neuquina provienen de unidades del Grupo Neuquén, fundamentalmente de las unidades superiores asignadas al Subgrupo Río Colorado, de las formaciones Anacleto y Bajo de la Carpa y también del subgrupo Río Limay, la Formación Candeleros. Las especies reconocidas son Notosuchus terrestris Woodward, 1896, Peirosaurus tormini Price, 1955, Lomasuchus palpebrosus Gasparaini, Chiappe \& Fernandes, 1991, Araripesuchus patagonicus Ortega et al., 2000, Comahuesuchus brachibuccalis Bonaparte, 1991 y Araripesuchus buitreraensis Pol \& Apesteguia, 2005 (Gasparini et al., 1991; Bonaparte, 1991; Martinelli, 2003; Pol \& Apesteguia, 2005). Estos Mesoeucrocodylia se incluyen actualmente al menos en dos clados: Notosuchia y Peirosauridae. Algunas de éstas especies han sido descriptas y estudiadas hace más de un siglo, y desde mediados del siglo XX su diversidad se ha visto incrementada, y sus re- 
laciones de parentesco más resueltas gracias a la aplicación de la sistemática filogenética.

En la zona de la localidad de Rincón de los Sauces, situada en el noroeste de la Provincia de Neuquén y a orillas del Río Colorado, límite natural con la Provincia de Mendoza, afloran unidades del Cretácico Superior de la Cuenca Neuquina (Figura 1). En diferentes sitios de esta área, conocidos como Loma del Lindero, Cañadón Arroyo Río Seco, Puesto Hernández y Narambuena, se han hallado abundantes restos de vertebrados que incluyen varias formas de saurópodos titanosaurios, decenas de dientes de terópodos y restos de tortugas. En este trabajo se describe un ejemplar de crocodiliforme encontrado el último sitio mencionado $\left(37^{\circ} 24^{\prime} 40^{\prime \prime} \mathrm{S}, 6^{\circ} 05^{\prime} 46^{\prime \prime} \mathrm{O}\right)$, durante excavaciones realizadas en el año 2000 por una comisión dirigida por uno de los autores (J.C.). Se trata de un espécimen incompleto que incluye parte del postcráneo articulado de un individuo posiblemente subadulto, debido a la falta de fusión entre arco neural y cuerpo vertebral en la zona dorsal y a su pequeño tamaño, de una longitud total estimada en $60 \mathrm{~cm}$.

Los estudios anatómicos y filogenéticos acerca de los Crocodyliformes se basan fundamentalmente en materiales craneanos. Esto puede deberse a que restos postcraneales desarticulados son en general difíciles de asignar a un taxón concreto o quizás, porque que se ha considerado que el esqueleto postcraneal de los cocodrilos ha sido evolutivamente

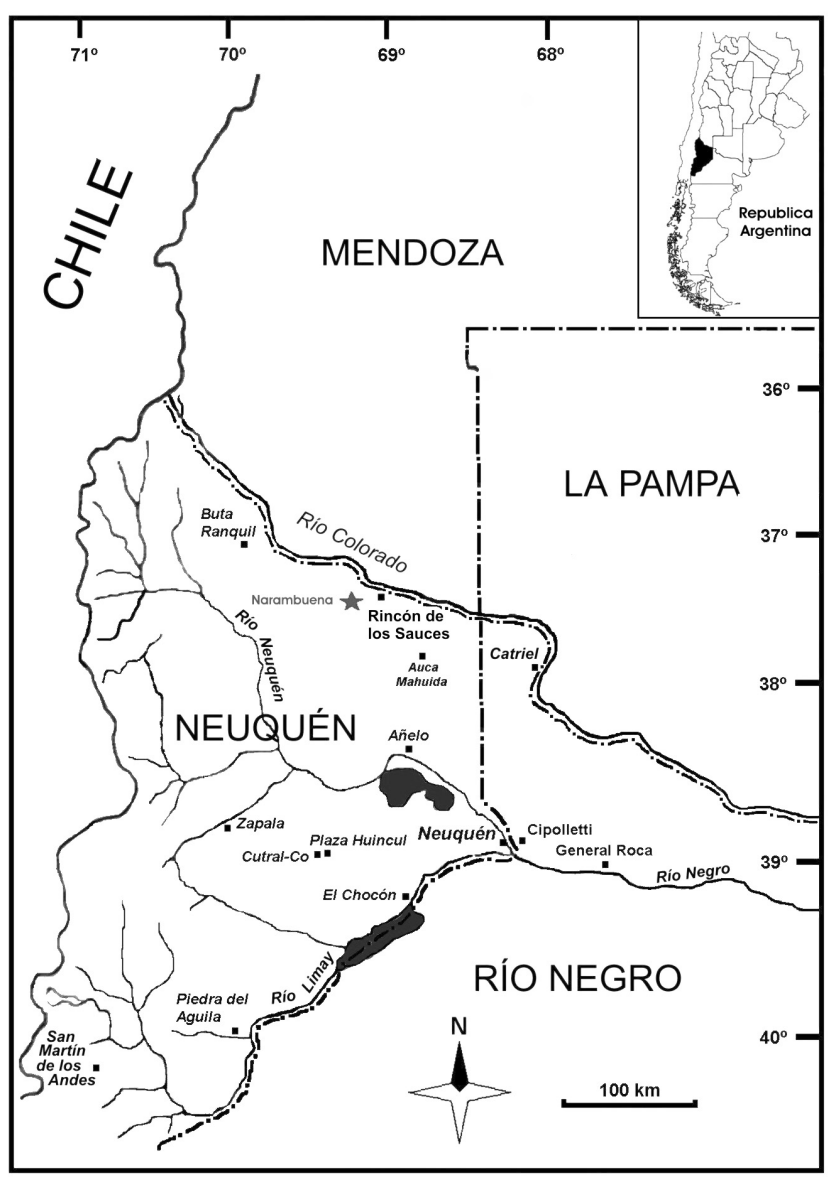

Figura 1. Mapa de ubicación de la localidad fosilífera.

Figure 1. Map with the location of the fossiliferous site. "conservador". De esta manera, la anatomía postcraneana de las especies de cocodrilos cretácicos ha permanecido postergada en su análisis, como ya ha sido notado por varios autores (Pol, 1999a,b). Más recientemente, sin embargo, algunos caracteres postcraneanos han sido incluidos en propuestas filogenéticas y se ha puesto de manifiesto su valor taxonómico y filogenético (Pol, 1999 a,b,c, 2003; Ortega et al., 2000; Sereno et al., 2003; Carvalho et al., 2004; Turner, 2006) en contra de hipótesis previas que no incluían caracteres postcraneanos en las hipótesis filogenéticas propuestas para este grupo (Langston, 1975; Clark, 1994).

Debido a que este ejemplar es uno de los pocos restos de Crocodyliformes hallados en el extremo norte de la Cuenca Neuquina, se realizó el estudio del mismo con el objeto de compararlo con los Mesoeucrocodylia (Notosuchia y Peirosáuridos) previamente descritos en el resto de la cuenca (Gasparini et al., 1991; Bonaparte, 1991; Ortega et al., 2000; Leanza et al., 2004; Candeiro et al., 2006; Pol \& Apesteguia, 2005; Pol \& Gasparini, 2007). A pesar la naturaleza fragmentaria de este crocodiliforme, este estudio se llevó a cabo ya que consideramos que el ejemplar posee caracteres relevantes desde el punto de vista taxonómico. No se lo ha incluido, sin embargo, en una matriz filogenética debido a que consideramos que la ausencia total de caracteres craneanos, que son mayoría en los taxones comparables, no permitiría obtener resultados congruentes.

El ejemplar que aquí se describe esta semiarticulado, y es posiblemente un subadulto debido a la falta de fusión entre arco neural y cuerpo en algunas vértebras y por su pequeño tamaño. Está compuesto por una serie vertebral torácicaslumbar-caudal casi completa y articulada, así como los miembros posteriores, estrechamente asociados a la serie vertebral, escápula e ilion; con el cráneo, el sector cervical y gran parte de los elementos distales manuales y pediales ausentes. El material se encuentra depositado en Museo Municipal Argentino Urquiza, Narambuena, Rincón de los Sauces, Neuquén. Abreviatura institucional. MAU-Pv-N, Museo Municipal Argentino Urquiza, Paleontología de Vertebrados, Narambuena, Rincón de los Sauces, Neuquén.

\section{MARCO GEOLÓGICO}

Los restos estudiados proceden del Yacimiento Petrolífero Narambuena ubicado a unos $15 \mathrm{~km}$ al oeste de la ciudad de Rincón de los Sauces. En esta región afloran sedimentitas correspondientes a las Formaciones Portezuelo y Plottier que conforman la parte media del Grupo Neuquén (Figura 2). Este grupo esta dividido en tres Subgrupos: Río Limay, el más inferior, Río Neuquén el intermedio y Río Colorado, el más superior (Leanza et al., 2004). Estas secuencias se interpretan como depositadas en un conjunto muy variado de ambientes: fluvial, aluvial, eólico y de playa. Al oeste de la localidad de Rincón de los Sauces, afloran sobrepuestos a la Formación Portezuelo, una estrecha faja de estratos rojos asignados a la Formación Plottier por varios autores, como Fossa Mancini et al. (1938), Digregorio (1972), Cazau \& Uliana (1973), Legarreta \& Gulisano (1989), Legarreta \& Uliana (1999), 


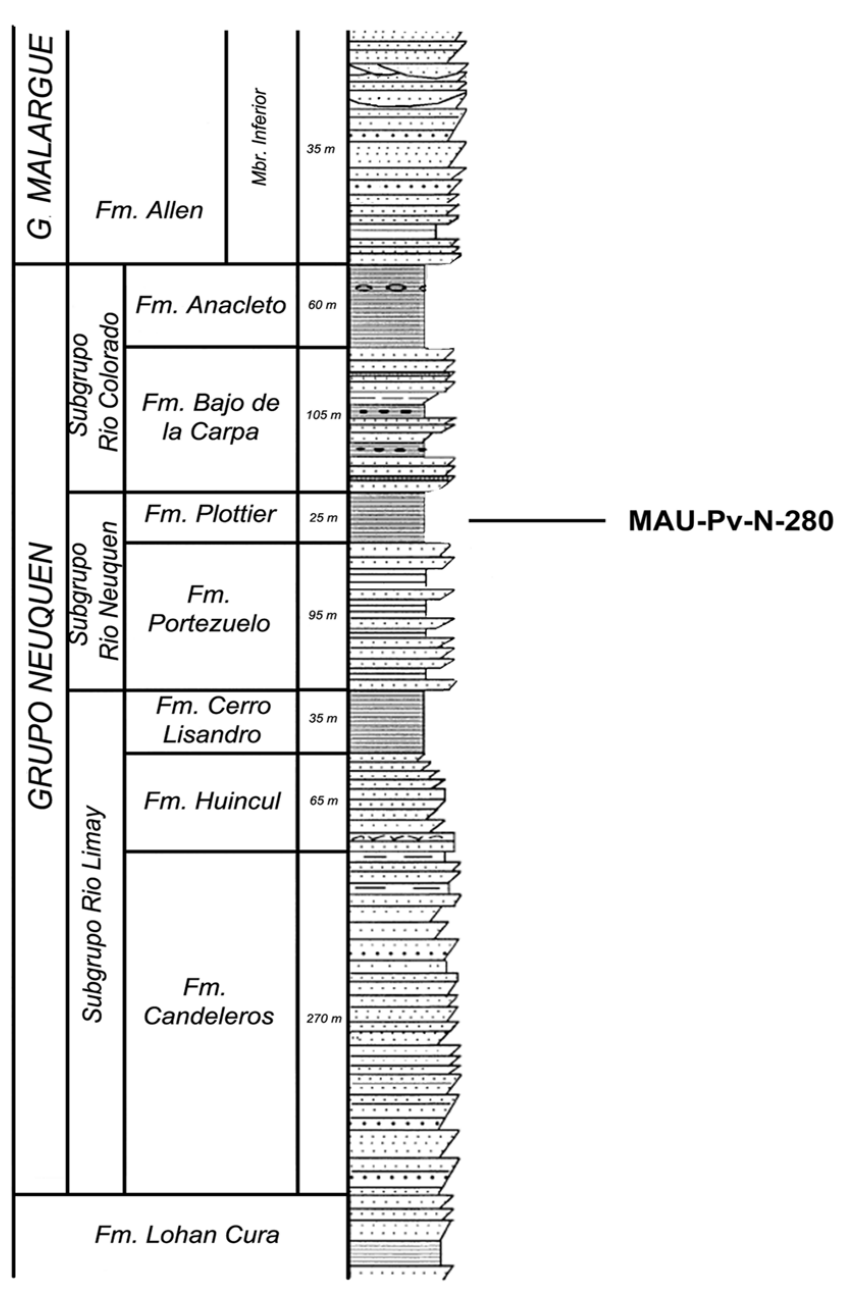

Figura 2. Columna mostrando la posición estratigráfica del espécimen de Mesoeucrocodylia indet. (MAU-Pv-N-280), Narambuena, Formación Plottier (modificada de Leanza et al., 2004).

Figure 2. Stratigraphic section showing the location of the studied specimen Mesoeucrocodylia indet. (MAU-Pv-N-280), Narambuena, Plottier Formation (modified from Leanza et al., 2004).

Leanza \& Hugo (2001), Sanchez et al. (2006), entre otros. La Formación Plottier fue definida por Herrero Ducloux (en Fossa Mancini et al., 1938). La localidad tipo está al Norte de la localidad de Plottier cerca del Aeropuerto de la Ciudad de Neuquén. Sus niveles están sobrepuestos en conformidad a la Formación Portezuelo, con la cual se intercalan lateralmente y está cubierta a su vez por niveles de la Formación Bajo de la Carpa. La Formación Plottier es, en general, difícil de distinguir en el campo, y la principal diferencia con la subyacente Formación Portezuelo es la mayor proporción de contenido arcilloso (Leanza et al., 2004). Está compuesto por arcillitas masivas rojo claro con finas capas de lutitas rosadas. Su máximo espesor es de alrededor de $25 \mathrm{~m}$ y su edad se considera Coniaciano superior (Leanza \& Hugo, 2001; Leanza et al., 2004).

La sección estudiada se compone de $2 \mathrm{~m}$ de pelitas pardas sin detalle de laminación. Hacia el tope de este banco se hallaron los restos del crocodiliforme estudiado. Luego hay $0,50 \mathrm{~m}$ de areniscas conglomeráticas polimícticas de color gris claro. Los clastos son en su mayor parte de arcillitas de hasta $1 \mathrm{~cm}$ de diámetro y fragmentos de huesos de vertebrados transportados. El cemento que aglutina los clastos y la matriz son calcáreos. Luego hay $1,50 \mathrm{~m}$ de una arenisca friable de color rojizo sin detalle de estructuras, $4,10 \mathrm{~m}$ de pelitas de color rojo pardo y $0,40 \mathrm{~m}$ de arenisca grueso de color gris rojizo deleznable, con algunos clastos de hasta $0,5 \mathrm{~mm}$ de diámetro. Por encima hay 3,20 m de pelitas de color marrón y se desarrollan nódulos de hasta $20 \mathrm{~cm}$ de largo de calcita de origen secundario, como así también niveles de yeso de origen secundario. La secuencia se ve interrumpida por pelitas color verde claro de no más de $10 \mathrm{~cm}$ de grosor. Siguen $0,30 \mathrm{~m}$ de conglomerado compuesto de clastos pelíticos de color rojizo y $6,60 \mathrm{~m}$ de arenisca mediana a gruesa de color gris claro con algunos bancos de estratificación entrecruzada en artesa, indicando un origen fluvial de alta energía.

\section{SISTEMÁTICA PALEONTOLÓGICA}

\author{
ARCHOSAURIA Cope, 1869 \\ CROCODYLOMORPHA Hay, 1930 (sensu Walker, 1970) \\ CROCODYLIFORMES Hay, 1930 (sensu Clark, 1986) \\ MESOEUCROCODYLIA Whetstone y Whybrow, 1983 \\ MESOEUCROCODYLIA indet.
}

(Figuras 3-6; Tabla 1)

Material. MAU-PV-N-280, fémur izquierdo, tibia y fíbula izquierdas articuladas; fémur derecho incompleto, tibia y fíbula derechas con astrágalo y calcáneo articulados; serie caudal articulada de 21 vértebras. C3, C4 y C5 en la serie articuladas a los arcos hemales; serie dorsal parcialmente articulada de 11 vértebras; escápula izquierda; ilion izquierdo; porciones proximales de los isquiones?; porción proximal de húmero?; un metatarso incompleto, 10 osteodermos, 9 articulados y asociadas a las vértebras caudales, uno aislado e incompleto y otros fragmentos indeterminados.

Procedencia geográfica. Coordenadas $37^{\circ} 24^{\prime} 40^{*} \mathrm{~S} /$ $69^{\circ} 05^{\prime} 46^{\circ} \mathrm{O}$, Yacimiento Narambuena, aproximadamente a unos 15 km oeste de Rincón de los Sauces, Provincia de Neuquén, Argentina.

Horizonte estratigráfico. Grupo Neuquén, Subgrupo Río Neuquén, Formación Plottier.

Edad. Cretácico Superior (Coniaciano superior).

Diagnosis. Mesoeucrocodylia con fémur sigmoideo, proceso postacetabular del ilion: muy corto, sin lámina en el borde dorsal del ilion, ni cresta supracetabular desarrolladas. Dos filas paramedianas de osteodermos, con cresta dorsal ubicada en el centro de la superficie y con ornamentación en forma de surcos y crestas poco definidas que se extienden radialmente desde la cresta hacia los bordes.

English diagnosis. Mesoeucrocodylia with a sigmoidal femur, a postacetabular process of ilium very short, without a plate at the dorsal margin, and without developed supracetabular crest. Two row of parasagital osteoderms with a dorsal crest at the midline of surface and ornamentation formed by sulci and discrete crests, extending radially from the central crest to the margins.

Descripción. Esqueleto axial: (i) vértebras torácicas-lumbares 
- las vértebras conservadas corresponden a las siete últimas torácicas y cuatro lumbares. Los cuerpos vertebrales son anficélicos, con su borde ventral cóncavo y sus caras laterales excavadas. Los procesos transversos están mal conservados, pero su base se encuentra ubicada muy dorsalmente en la cara lateral del arco neural. Las vértebras dorsales más anteriores preservadas se hallan en su mayoría con el arco neural desarticulado del centro vertebral, lo que indica la condición juvenil del ejemplar. Las vértebras lumbares muestran las suturas entre el arco neural y el cuerpo lo que podría estar relacionado con la condición subadulta del especímen (Brochu, 1996); (ii) vértebras caudales - la serie caudal se encuentra casi completa y consta de 21 vértebras, de un total de probablemente 25. Presentan cuerpos vertebrales fusionados a los arcos neurales. Los cuerpos de las caudales son más cortos craniocaudalmente que los cuerpos de las dorsales posteriores. Los procesos transversos se encuentran en la base de los arcos neurales. En las caudales proximales las espinas neurales están preservadas, son altas y angostas anteroposteriormente, constituyendo aproximadamente dos veces la altura del cuerpo vertebral. A partir de la segunda vértebra caudal se observan los procesos de articulación para los arcos hemales. Estos arcos están conservados en varias de las caudales proximales, son rectos y comprimidos lateralmente, están proyectados posteriormente y su longitud es comparable a la de las espinas neurales. Las caudales proximales presentan escudos dorsales articulados a ambos lados de las espinas neurales, correspondiendo un par de escudos a cada vértebra. Las caudales distales tienen los cuerpos mas alargados, y en ellas disminuye progresivamente la altura de las espinas neurales. Las postzigapófisis de las caudales proximales no superan el borde del cuerpo vertebral, mientras que en las caudales distales son más alargadas (Figura 3).

Esqueleto apendicular: (i) escápula - la escápula corresponde al lado izquierdo y tiene la forma característica de los crocodilomorfos, con un área constreñida que divide la lámina dorsal de una expansión ventral que posee la parte dorsal de la fosa glenoidea. La hoja escapular es convexa en su cara lateral y cóncava en su cara medial. La extensión total de la lámina de la escápula es imposible de medir en el ejemplar debido a que no se ha preservado el borde anterodorsal de dicha lámina, así como el borde distal. En el borde posteroventral presenta una cresta longitudinal que correspondería a la inserción del musculus serratus superficialis (Turner, 2006). Ventralmente la escápula se expande en una plataforma redondeada que contacta con el coracoides y forma la parte dorsal de la cavidad glenoidea. Por el estado de preservación no es posible observa la presencia de un proceso acromial; (ii) ilión - el ilion izquierdo presenta un borde dorsal convexo, robusto, con escasa expansión del proceso preacetabular. El proceso postacetabular está incompleto y en una posición más dorsal que el acetábulo. La porción dorsal de la hoja ilíaca muy desarrollada en otros crocodilomorfos como Araripesuchus tsanganatsangana Turner, 2006, está ausente en esta forma de Rincón de los Sauces, y también en Notosuchus terrestris Woodward, 1896 (Pol, 2005). El acetábulo es amplio y abierto, sin rebordes óseos que lo delimiten, con la cresta supracetabular poco desarrollada. El pedicelo isquiático está conservado, es robusto y está proyectado lateroventralmente (Figura 4); (iii) fémur - se ha conservado un fémur izquierdo completo y uno derecho incompleto. La diáfisis está comprimida transversalmente, probablemente por deformación, y es sigmoidea en vista lateral, condición muy diferente a la de los fémures rectos que presentan los notosuchia (Pol, 2003). Los fémures de Araripesuchus son algo más sigmoideos que los de Notosuchus, aunque no presentan la condición derivada, típica de los Neosuchia. La diáfisis es relativamente robusta, en comparación con los de otros Notosuquia (Turner, 2006). La cabeza femoral está ligeramente aplastada, por deformación postmortem. El área correspondiente al trocánter cuarto esta algo dañada. El trocánter cuarto no está bien desarrollado, y en su lugar hay una conspicua cicatriz, a di-

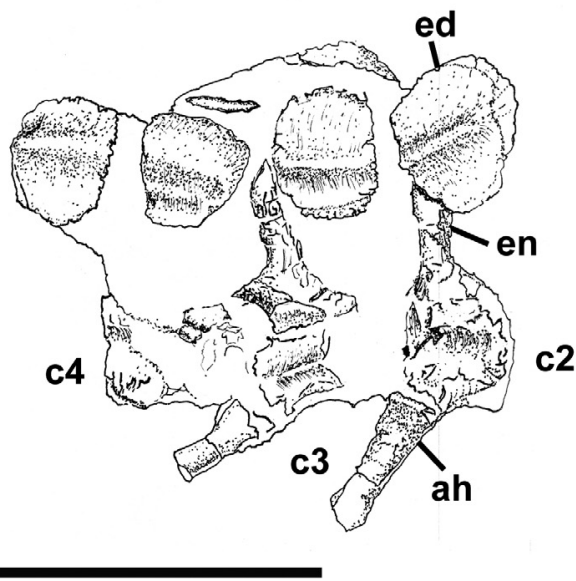

Figura 3. Mesoeucrocodylia indet. (MAU-Pv-N-280), Narambuena, Formación Plottier. Vista lateral de vértebras caudales articuladas con arcos hemales y escudos dérmicos articulados. Abreviaturas: ah, arco hemal; ed, escudo dérmico; en, espina neural; c2-c4, caudales 2-4. Escala $=50 \mathrm{~mm}$.

Figure 3. Mesoeucrocodylia indet. (MAU-Pv-N-280), Narambuena, Plottier Formation. Lateral view of articulated caudal vertebrae with hemal arches and dorsal dermal scutes articulated in place. Abbreviations: ah, hemal arches; ed, dermal scutes; en, neural spine; c2c4, caudals two to four. Scale bar $=50 \mathrm{~mm}$. 
ferencia de lo observado en otros Mesoeucrocodylia como Notosuchus, Mahajangasuchus y Araripesuchus (Pol, 2005). En vista lateral se puede observar que la cabeza esta ligeramente orientada anteriormente. El extremo distal del fémur es robusto y está expandido transversalmente. El cóndilo lateral es globoso y ocupa la mayor parte del extremo distal. El cóndilo medial está muy aplastado transversalmente. En vista anterior, el extremo distal presenta un surco intercondilar anterior muy somero, mientras que el surco intercondilar posterior es muy profundo (Figura 5).

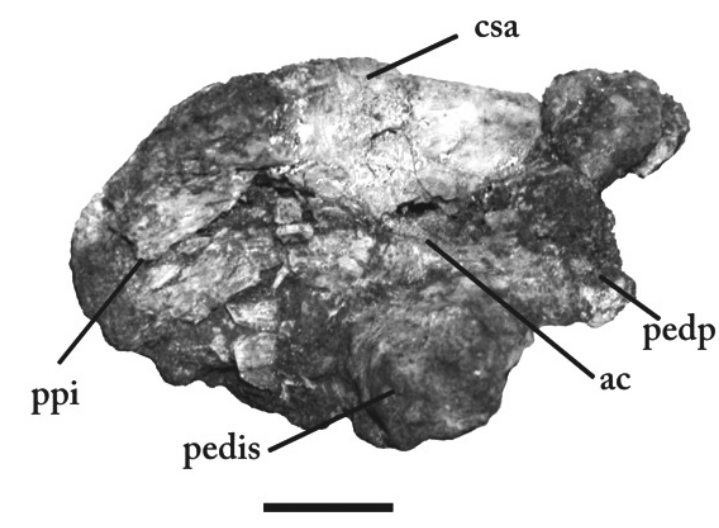

Figura 4. Mesoeucrocodylia indet. (MAU-Pv-N-280), Narambuena, Formación Plottier. Vista lateral ilion izquierdo. Abreviaturas: ac, acetábulo; csa, cresta supracetabular; pedis, pedicelo isquiático; pedp, pedicelo púbico; ppi, proceso posterior del ilion. Escala $=10 \mathrm{~mm}$.

Figure 4. Mesoeucrocodylia indet. (MAU-Pv-N-280), Narambuena, Plottier Formation. Lateral view of left ilium. Abbreviations: ac, acetabulum; csa, supraacetabular crest; pedis, ischiatic pedicel; pedp, pubic pedicel; ppi, posterior process of ilium. Scale bar $=10 \mathrm{~mm}$.
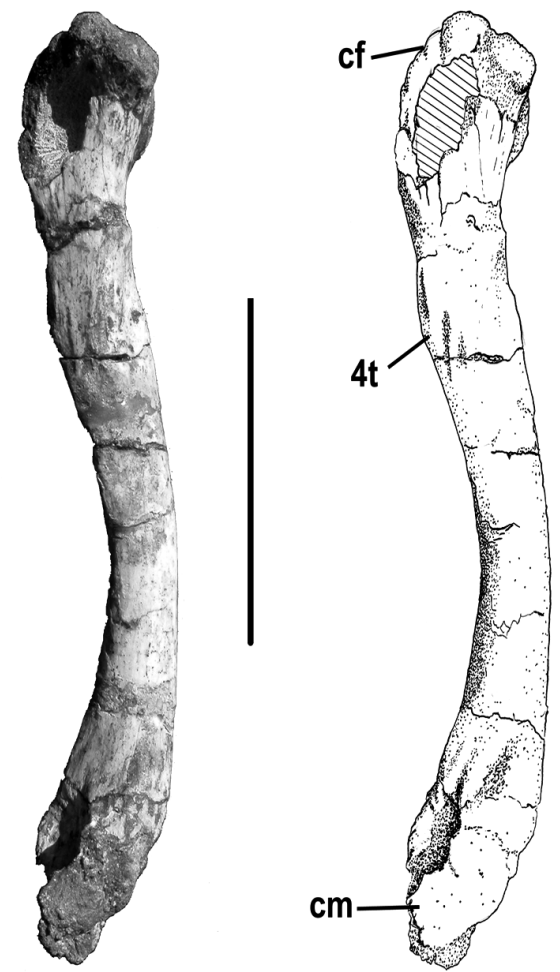

Figura 5. Mesoeucrocodylia indet. (MAU-Pv-N-280), Narambuena, Formación Plottier. Vista lateral fémur izquierdo. Abreviaturas: cf, cabeza femoral; cm, cóndilo medial; 4t, cuarto trocánter. Esca$\mathrm{la}=50 \mathrm{~mm}$.

Figure 5. Mesoeucrocodylia indet. (MAU-Pv-N-280), Narambuena, Plottier Formation. Lateral view of left femur. Abbreviations: cf femoral head; $\mathbf{c m}$, medial condile; $\mathbf{4 t}$, fourth trocanter. Scale bar $=$ $50 \mathrm{~mm}$.

Tabla 1. Medidas del espécimen de Mesoeucrocodylia indet. (MAU-Pv-N-280), Narambuena, Formación Plottier. Abreviaturas: L, longitud; ALD, ancho lateral del diáfisis; AAD, ancho anteroposterior del diáfisis; LMe, longitud menor; LMa, longitud mayor; LC, longitud centro; AC, altura centro; AAP, ancho anteroposterior; $\mathbf{A L}$, ancho lateral; $\mathbf{m m}$, milímetros.

Table 1. Measurements of the studied specimen Mesoeucrocodylia indet. (MAU-Pv-N-280), Narambuena, Plottier Formation. Abbreviations: L, length; ALD, diaphysis lateral width; AAD, diaphysis anteroposterior width; LMe, lesser length; LMa, greater length; LC, centrum length; AC, centrum heigth; AAP, anteroposterior width; $\mathbf{A L}$, lateral width; $\mathbf{m m}$, millimiters.

\begin{tabular}{|c|c|c|c|c|c|c|c|c|c|}
\hline Material & $\begin{array}{c}\mathrm{L} \\
(\mathrm{mm})\end{array}$ & $\begin{array}{l}\text { ALD } \\
(\mathrm{mm})\end{array}$ & $\begin{array}{l}\text { AAD } \\
(\mathrm{mm})\end{array}$ & $\begin{array}{l}\mathrm{LMe} \\
(\mathrm{mm})\end{array}$ & $\begin{array}{l}\mathrm{LMa} \\
(\mathrm{mm})\end{array}$ & $\begin{array}{c}\mathrm{LC} \\
(\mathrm{mm})\end{array}$ & $\begin{array}{c}\mathrm{AC} \\
(\mathrm{mm})\end{array}$ & $\begin{array}{l}\text { AAP } \\
(\mathrm{mm})\end{array}$ & $\begin{array}{c}\mathrm{AL} \\
(\mathrm{mm})\end{array}$ \\
\hline Fémur izquierdo & 128 & 10,3 & 14,2 & & & & & & \\
\hline Tibia derecha & 116 & 8,5 & 10,3 & & & & & & \\
\hline *Fíbula derecha & $* 96,9$ & 5,0 & 5,5 & & & & & & \\
\hline **Tibia izquierda & 105,5 & $* * 11,3$ & $* * 7,8$ & & & & & & \\
\hline Fíbula izquierda & 101,8 & 5,0 & 5,5 & & & & & & \\
\hline Ilión izquierdo? & & & & 28,8 & 47,8 & & & & \\
\hline Centro cervical? & & & & & & 16,4 & 10,8 & & \\
\hline Centro torácico & & & & & & 17,2 & 11,0 & & \\
\hline Centro lumbar & & & & & & 18,6 & 12,8 & & \\
\hline $1^{\circ}$ Centro caudal & & & & & & 13,0 & 7,3 & & \\
\hline $3^{\circ}$ Centro caudal & & & & & & 14,3 & 8,4 & & \\
\hline $4^{\circ}$ Centro caudal & & & & & & 14,3 & 8,4 & & \\
\hline $5^{\circ}$ Centro caudal & & & & & & 14,1 & 7,8 & & \\
\hline Centro caudal medio & & & & & & 11,0 & 4,8 & & \\
\hline Centro caudal distal & & & & & & 9,0 & 4,3 & & \\
\hline Escudo dérmico dorsal & & & & & & & & 12,8 & 17,3 \\
\hline
\end{tabular}


Tibia: ambas tibias están completas y articuladas con las fíbulas. Su longitud es aproximadamente es un $10 \%$ menor a la longitud del fémur. El extremo proximal de la tibia es robusto, ligeramente mas expandido transversalmente que anteroposteriormente. Los dos cóndilos tibiales están bien diferenciados por una concavidad medial. El cóndilo proximal medial es más bajo que el cóndilo lateral, configurando una articulación escalonada con los cóndilos femorales, lo que también está presente en Araripesuchus patagonicus Ortega et al. 2000. La diáfisis es esbelta, y posee una sección transversal triangular y está curvada anteriormente. El extremo distal está expandido transversalmente, y presenta una cara anterior plana (Figura 6); (iv) fíbula - las fíbulas se han conservado en ambos casos articuladas con las tibias. Son huesos esbeltos, aproximadamente de un diámetro un 50\% menor que las tibias. El extremo anterior está expandido anteroposteriormente, firmemente articulado sobre la cara lateral del cóndilo tibial

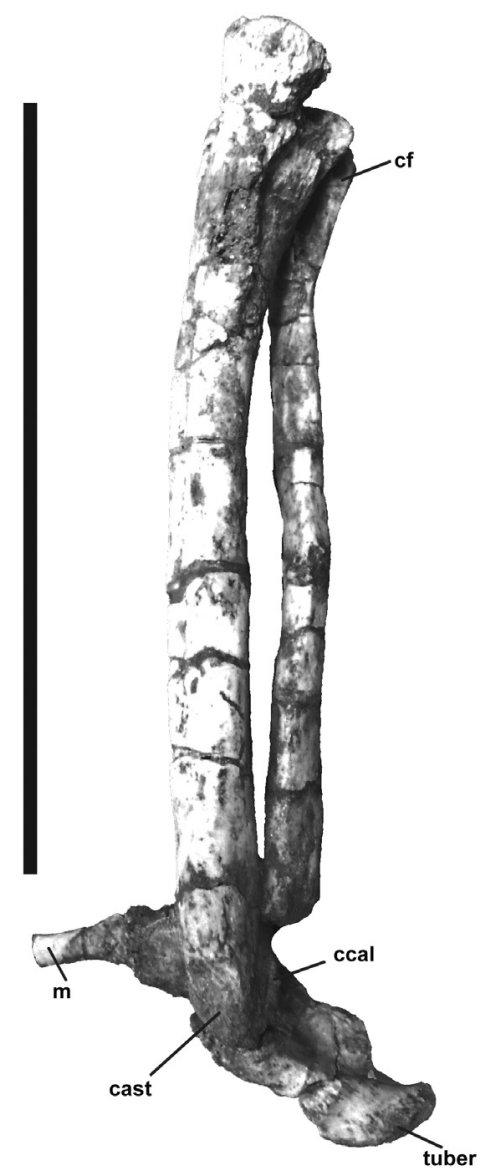

Figura 6. Mesoeucrocodylia indet. (MAU-Pv-N-280), Narambuena, Formación Plottier. Vista posterolateral de tibia y fíbula articuladas al astrágalo calcáneo. Abreviaturas: cf, cresta fibular; m, metatarso; tuber, túber del calcáneo; cast, cóndilo del astrágalo; ccal, cóndilo del calcáneo. Escala $=100 \mathrm{~mm}$.

Figure 6. Mesoeucrocodylia indet. (MAU-Pv-N-280), Narambuena, Plottier Formation. Lateroposterior view of articulated tibia-fibula and astragalus-calcaneum. Abbreviations: cf, fibular crest; m, metatarsal; tuber, calcaneal tuber; cast, astragalar condyle; ccal, calcaneal condyle. Scale bar $=100 \mathrm{~mm}$. externo. El borde posterior del extremo proximal presenta un marcado proceso óseo. El extremo distal esta expandido transversalmente en forma laminar y superpuesto sobre la cara anterior del extremo distal de la tibia; (v) astrágalo-calcáneo - los tarsales proximales están mal preservados, ya que la superficie del hueso se encuentra meteorizada. La morfología tarsal corresponde a la condición crocodilomorfa basal denominada "cocodriloideo normal" (Sereno \& Arcucci, 1990), que permite un movimiento de rotación entre ambos huesos. Estos huesos están articulados entre sí, no fusionados, y articulados a la tibia izquierda, aunque no en su posición anatómica original. El astrágalo es cuadrangular, típicamente cocodriliano, con un proceso ascendente poco desarrollado dorsalmente y una depresión en la cara anterior. El calcáneo tiene cuerpo cuadrangular, posee un tubérculo muy desarrollado de forma redondeada que se proyecta posterolateralmente y esta separado del cuerpo del hueso por una escotadura dorsal (Figura 6). Esta condición difiere de la observada en Araripesuchus tsanganatsangana Turner, 2006, donde el túber del calcáneo esta delimitado dorsal y centralmente por dos escotaduras que forman un "cuello".

Armadura dérmica: (i) osteodermos - se ha conservado los osteodermos de la región caudal asociados a las vértebras (Figura 3). Son pares, dispuestos en filas y tienen forma cuadrangular a oval. Poseen una quilla longitudinal dorsal central, baja y robusta. Carecen de procesos articulares o de regiones marginales para su articulación con otros osteodermos. La ornamentación es poco marcada y no está bien conservada, pero consiste en surcos o estrías, disponiéndose radialmente a partir de la cresta central. No presenta fosetas. No se han hallado osteodermos ventrales ni escudos asociados a los miembros.

\section{DISCUSIÓN}

Mientras que los cocodrilos más antiguos de este período en América del Sur provienen del Cretácico temprano de Brasil (Buffetaut, 1994) y Uruguay, en Argentina se conocen desde el Cretácico Superior temprano (Cenomaniano) hasta el Cretácico más terminal (Ortega et al., 2000; Leanza et al., 2004; Pol \& Apesteguia, 2005; Candeiro \& Martinelli, 2006; Candeiro et al., 2006). En África también están registrados durante todo este período, desde el Cretácico Inferior temprano de Níger hasta el Campaniano Maastrichtiano en Madagascar (Turner, 2004, 2006; Larsson \& Sues, 2007; Sereno \& Larsson, 2009).

Los Mesoeucrocodylia más antiguos de la Cuenca Neuquina son formas cercanas al género Araripesuchus, registrados en la Formación Candeleros del subgrupo Rio Limay (Leanza et al., 2004). Tradicionalmente ha habido una controversia acerca de su posición filogenética debido a la combinación de caracteres primitivos y derivados que presentan. Las diferencias entre los análisis realizados se 
deben en parte a diferencias en los datos y a los taxones empleados en cada trabajo. Araripesuchus fue tradicionalmente considerado relacionado a Notosuchus y otras formas cretácicas sudamericanas, aunque en análisis cladísticos recientes aparece en diferentes posiciones dentro de los clados más basales de Mesoeucrocodylia (Wu \& Sues, 1996; Buckley et al., 2000; Ortega et al., 2000; Pol, 2003; Sereno et al., 2003; Pol \& Apesteguia, 2005; Turner, 2006). Se pueden distinguir dos posiciones básicamente contradictorias (y no resueltas aún) acerca de su relación filogenética con otros Mesoeucrocodylia: algunos análisis muestran a Araripesuchus mas cerca de los Notosuchia que de los Neosuchia (Pol \& Norell, 2004; Pol \& Apesteguia, 2005) mientras otros los muestran como grupo hermano de los neosuquios que de los notosuquios (Ortega et al., 2000; Buckley et al., 2000; Turner \& Calvo, 2005). Asimismo subsisten preguntas acerca de la monofilia de los araripesúquidos, debido principalmente a la dentición zifodonta, tan diferente a los demás formas, que presenta A. wegeneri Buffetaut, 1981 (discusión in Turner, 2006), y de hecho tanto éste como A. buitreraensis Pol \& Apesteguia, 2005, podrían ser formas más cercanas a otros notosuquios, como Simosuchus o Anatosuchus que a otros representantes del género (Sereno \& Larsson, 2009).

Los Notosuchia encontrados en esta cuenca se localizan en las cercanías de la ciudad de Neuquén, en niveles del Grupo Neuquén, ahora considerados dentro del subgrupo Río Colorado (Leanza et al., 2004) en niveles asignados a la formación Bajo de la Carpa (SantonianoCampaniano) (Gasparini, 1971, 1981; Pol \& Gasparini, 2007), se halló una importante colección de cocodrilos brevirostros referidos por Woodward (1896) a la nueva especie Notosuchus terrestris Woodward, 1896. Los notosuquios tuvieron una amplia distribución geográfica en América del Sur. Están presentes en el Cretácico tardío en el noroeste de Uruguay (Uruguaysuchus aznarezi y Uruguaysuchus terrai, ambos descriptos por Rusconi, 1933), en Brasil Candidodon (Carvalho et al., 2004) y en Argentina (N. terrestris y Comahuesuchus brachibucalis, Bonaparte, 1991). Algunos caracteres anatómicos de los Notosuchia tales como rostro corto, pocos dientes cuspidados, una cierta heterodoncia, y las narinas externas de posición terminal, pero sobre todo la información que aporta el esqueleto postcraneal, indican que probablemente eran cocodrilos de hábitos más terrestres que los actuales.

Price (1955) describe un nuevo crocodilia, Peirosaurus tormini, del Senoniano de la Formación Bauru (Campaniano?/Maastrichtiano o Maastrichtiano) del sudeste de Brasil, incluyéndolo dentro de Sebecosuchia. Los dientes con bordes aserrados, la forma de las narinas externas y la escultura vermiforme fueron las primeras características citadas en el soporte de esta asignación (Gasparini et al., 1991). Más tarde, Romer (1956) incluye Peirosaurus en los Sebecidae, mientras que Langston
(1975) y Bonaparte (1978) lo asignaron a Baurusuchidae, la otra familia de sebecosúquidos. Sin embargo Bonaparte (1978) sugiere que, en relación con los dientes aserrados, puede estar relacionados a los uruguaysúquios notosuquios (Gasparini et al., 1991). Buffetaut (1982) confirma la idea de Bonaparte, incluyendo a Peirosaurus dentro de Uruguaysuchidae, a pesar de que recientemente lo asignó a los Trematochampsidae (Gasparini et al., 1991). Sobre la base de un espécimen de $P$. tormini, del Cetácico Superior de la Subgrupo Río Colorado, Formación Bajo de la Carpa en el noroeste de Patagonia (Loma de la Lata, Neuquén, Argentina). Gasparini (1981) reconoció la nueva familia Peirosauridae destacando sus remarcables diferencias con sebecosuquios y uruguaysuquios. Los peirosáuridos constituyen aparentemente un grupo monofilético distintivo. Hasta ahora los peirosáuridos han sido encontrados en Sudamérica (Argentina y Brasil) Lomasuchus y Peirosaurus de la Formación Bajo de la Carpa (Gasparini et al., 1991; Leanza et al., 2004), Peirosaurus tormini y Uberabasuchus de la Formación Bauru (Campaniano-Maestrichtiano) (Price, 1955; Carvalho et al., 2004) y probablemente Caririsuchus (Kellner, 1987). Recientemente en Africa han sido descriptas nuevas formas tanto en el Campaniano-Maestrichtiano de Madagascar como Mahangasuchus (Buckley \& Brochu, 1999) y en el continente, en la Formación Elrhaz (Aptiano, Niger) Stolokrosuchus lapparenti Larsson \& Gado, 2000. Stolokrosuchus tiene una relación como grupo hermano no resuelta con Peirosaurus and Lomasuchus, lo que soportaria la monofilia de Peirosauridae y su inclusión en ese clado.

Los notosúquidos, araripesúquidos, peirosáuridos y baurusúquidos, poseen una direfenciación morfológica bien marcada entre sí y aparentemente no constituyen un grupo monofilético, repartiéndose entre los dos linajes basales de Mesoeucrocodylia, pero todos ellos se apartan del bauplan que caracteriza a los Crocodylia de hábitos anfibios. Es una discusión que excede a este estudio si ese ha sido o no el plan corporal primitivo para los Crocodyliformes.

De acuerdo a la descripción realizada en este trabajo se advierte que los siguientes caracteres aportan evidencia preliminar sobre la identidad taxonómica y las afinidades filogenéticas del especímen de Rincón de los Sauces: (i) fémur sigmoideo - este carácter se ha propuesto como indicador de formas más derivadas de Mesoeucrocodylia, no se observa entre los notosúquidos, que poseen fémures rectos (con la excepción de algunos especimenes con fémures ligeramente sigmoideos en Araripesuchus); (ii) proceso postacetabular del ilión - muy corto en el ejemplar estudiado y se asemeja al estado presente en algunos Crocodyliformes neosuquianos; (iii) borde dorsal del ilion - la presencia de una lamina en el borde dorsal del ilion es un carácter plesiomórfico para los crocodyliformes, y no esta presente en el ejemplar estudiado, lo que relacionado 
con el carácter anterior vincularía al nuevo ejemplar con formas más derivadas que los Notosuchia. Tampoco se observa cresta supracetabular; (iv) número de filas de osteodermos dorsales - en el nuevo ejemplar hay dos filas de escudos paramedianas como en Araripesuchus patagonicus, y no cuatro o más como en algunos Neosuchia más derivados (Ortega et al., 2000); (v) forma de los osteodermos - los osteodermos dorsales caudales del nuevo ejemplar son de forma oval con su eje mayor dispuesto transversalmente. En notosuquios (incluyendo Araripesuchus) tienen una morfología muy similar, cuadrangulares sin proyecciones para la articulación entre placas; (vi) posición de la cresta dorsal de los osteodermos - en los araripesúchidos la cresta dorsal de los osteodermos está desplazada hacia el borde lateral, haciendo que el borde externo se proyecte lateralmente. En el ejemplar en estudio la cresta de los osteodermos esta bien desarrolada y se encuentra ubicada en el centro de la superficie dorsal. Es la misma condición presente en Araripesuchus wegeneri Buffetaut, 1981 y probablemente Notosuchus y Malawisuchus también comparten esa condición; (vii) ornamentación de los osteodermos dorsales - en el ejemplar en estudio las placas poseen una ornamentación superficial. Tiene surcos y crestas poco definidas que se extienden radialmente desde la cresta medial hacia los bordes, similar a la presente en osteodermos atribuidos a Notosuchus. En los araripesúchidos, si bien la ornamentación no es muy marcada tiene forma de fosetas de distribución irregular. Esta condición la compartirían Araripesuchus + Neosuchia. La distribución radial de la ornamentación en los osteodermos es más frecuente en formas juveniles (Ortega, com. pers.).

\section{CONCLUSIONES}

El estudio y las comparaciones realizadas en los restos utilizados en este trabajo aportan nueva información acerca de la variabilidad existente en los diferentes representantes de los Mesoeucrocodylia de la Cuenca Neuquina. Los restos postcraneanos estudiados poseen un conjunto de caracteres que constituirían una condición primitiva en este clado y una serie de sinapomorfías compartidas por los Neosuchia más basales. Estos caracteres se encuentran también en Araripesuchus y formas asociadas, y estos comparten a la vez sinapomorfías de Notosuchia y de la base de Neosuchia. Si bien esta problemática dificulta poder precisar la posición filogenética de este espécimen, demuestra claramente la necesidad de realizar análisis filogenéticos más detallados que incluyan caracteres postcraneanos.

El ejemplar de Rincón de los Sauces dado a conocer en este estudio exhibe caracteres que han sido atribuidos previamente a Notosuchia y a Neosuchia y lo que sugiere su pertenencia a un nuevo taxón, diferente a los previamente descriptos en esta cuenca. A pesar de que la asociación de los materiales es muy clara, se ha preferido no nominar un nuevo taxón basado en estos materiales hasta que sea posible contar con especímenes que permitan una descripción y diagnosis más completa.

\section{AGRADECIMIENTOS}

Al Municipio de Rincón de los Sauces, por facilitar la estadía en esa localidad de uno de los autores (A.B.A.), en dos oportunidades; a S. Palomo y A. Valenzuela del Museo Argentino Urquiza (Rincón de los Sauces, Neuquén) por su colaboración en los trabajos de campo y preparación del material. A los responsables de la colección de ese Museo por permitir el estudio del material. Al A. Kramarz por el acceso a material de consulta del la Colección del Museo Argentino de Ciencias Naturales "Bernardino Rivadavia". Al R. Coria (Museo Carmen Funes, Plaza Huincul) por facilitar el acceso a material inédito de ese museo, así como los medios técnicos para realizar fotografías y algunas de las ilustraciones utilizadas durante el estudio (a A.B.A. y L.F.). Los dibujos fueron realizados por M. Loza. Los paleontólogos D. Pol y F. Ortega aportaron opiniones sobre el tema y revisaron versiones previas de este manuscrito. Trabajos de campo financiados por Universidad Nacional del Comahue Proyecto I-155.

\section{REFERENCIAS}

Bonaparte, J.F. 1978. El Mesozoico de América del sur y sus tetrápodos. Opera Lilloana, 26:170.

Bonaparte, J.F. 1991. Los vertebrados fósiles de la Formación Rio Colorado, de la ciudad de Neuquén y sus cercanías, Cretácico Superior, Argentina. Revista del Museo Argentino de Ciencias Naturales “Bernardino Rivadavia”, Paleontología, 4:17-123.

Brochu, C.A. 1996. Closure of neurocentral sutures during crocodilian ontogeny: implications for maturity assessment in fossil archosaurs. Journal of Vertebrate Paleontology, 16:49-62.

Buckley, G.A. \& Brochu, C.A. 1999. An enigmatic new crocodile from the Upper Cretaceous of Madagascar. Special Papers in Palaeontolology, 60:149-175.

Buckley, G.A.; Brochu, C.A.; Krause, D. \& Pol, D. 2000. A pugnosed crocodyliform from the Late Cretacous of Madagascar. Nature, 405:941-944. doi:10.1038/35016061

Buffetaut, E. 1982. Radiation evolutive, paleoecologie et biogeographie des crocodiliens mesosuchiens. Mémoires de la Société géologique de France, 142:1-88.

Buffetaut, E.1994. A new crocodilian from the Cretaceous of Southern Morocco Comptes rendus de l'Académie des sciences, Sciences de la terre et des planets, Série 2, 319:1563-1568.

Calvo, J.O. \& Porfiri, J. 2010. New material of Peirosaurids from Neuquén, Patagonia: its age. Brazilian Geographical Journal, 1:50-64.

Candeiro, C.R.A. \& Martinelli, A.G. 2006. A review of paleogeographical and chronoestratigraphical distribution of mesoeucrocodylian species from the Upper Cretaceous beds from the Bauru (Brazil) and Neuquén (Argentina) groups, Southern South America. Journal of the South American Earth Science, 22:116-129. doi:10.1016/j.james.2006.08.001

Candeiro, C.R.A; Martinelli, A.G.; Ávilla, L. \& Rich, T. 2006. Tetrapods from the Upper Cretaceous (TuronianMaestrichtian) Bauru Group of Brazil: a reapraisal. Cretaceous Research, 27:923-946. doi: 10.1016/j.cretres.2006.05.002 
Carvalho, I.S.; Ribeiro, L.C. \& Ávila, L. 2004. Uberabasuchus terrificus sp. nov. A new Crocodylomorpha from the Bauru Basin (Upper Cretaceous) Brazil. Gondwana Research, 7(4):975-1002. doi:1016/S1342-937X(05)71079-0

Cazau, I.B. \& Uliana, M.A. 1973. El Cretácico Superior continental de la Cuenca Neuquina. In: CONGRESO GEOLÓGICO ARGENTINO, 5, 1973. Actas, Buenos Aires, 3:131-163.

Clark, J. 1994. Patterns of evolution in Mesozoic Crocodyliformes. In: N. Frazer \& H.D. Sues (eds.) In the shadows of the dinosaurs, Cambridge University Press, p. 84-97.

Digregorio, J. 1972. Neuquén. In: A.F. Leanza (ed.) Geología Regional Argentina, Academia Nacional de Ciencias, p. 439-505.

Fossa Mancini, E.; Feruglio, E. \& Yussen de Campana, J. 1938. Una reunión de geólogos de YPF y el problema de la terminología estratigráfica. Boletín de Informaciones Petroleras, 15:1-67.

Gasparini, Z. 1971. Los notosuchia del Cretácico de América del Sur como un nuevo infraorden de los Mesosuchia. Ameghiniana, 8(1):83-103.

Gasparini, Z. 1981. Los Crocodylia fósiles de la Argentina. Ameghiniana, 18(3-4):177-205.

Gasparini, Z.; Chiappe, L. \& Fernandez, M. 1991. A new Senonian peirosaurid (Crocodylomorpha) from Argentina and a synopsis of the South American Cretaceous crocodilians. Journal of Vertebrate Paleontology, 11(3):316-333.

Kellner, A.W. 1987. Ocorrência de um novo crocodiliano no Cretáceo inferior da Bacia do Araripe, Nordeste do Brasil. Anais da Academia Brasileira de Ciências, 59(3):219-232.

Langston, W. 1975. Ziphodont crocodiles: Pristichampsus vorax (Troxell) new combination, from the Eocene of North America. Fieldiana Geology, 33:291-314.

Larsson, H.C.E. \& Gado, B. 2000. A new Early Cretaceous crocodyliform from Niger. Neues Jahrbuch für Mineralogie, Geologie und Paläontologie, 217:131-141.

Larsson, H.C.E. \& Sues, H-D. 2007. Cranial osteology and phylogenetic relationships of Hamadasuchus rebouli (Crocodyliformes: Mesoeucrocodylia) from the Cretaceous of Morocco. Zoological Journal of the Linnean Society, 149:53567. doi: 10.1111/j.1096-3642.2007.00271.x

Leanza, H. \& Hugo, C.A. 2001. Cretaceous red beds from southern Neuquen Basin (Argentina): age, distribution and stratigraphic discontinuities. In: INTERNATIONAL SYMPOSIUM OF MESOZOIC TERRESTRIAL ECOSYSTEMS, 7, 2001. Publicación especial, Buenos Aires, APA, p. 117-122.

Leanza, H.; Apesteguia, S.; Novas, F. \& De La Fuente, M. 2004. Cretaceous terrestrial beds from the Neuquén Basin (Argentina) and their tetrapod assemblages. Cretaceous Research, 25:61-87.

Legarreta, L. \& Gulisano, C.A. 1989. Análisis estratigráfico de la Cuenca Neuquina (Triásico Superior-Terciario inferior, Argentina). In: G. Chebli \& L. Spalletti (eds.) Cuencas Sedimentarias Argentinas: Serie Correlación Geológica, Universidad Nacional de Tucumán, 6:221-244.

Legarreta, L. \& Uliana, M. 1999. El Jurásico y Cretácico de la Cordillera Principal y Cuenca Neuquina. 1. Facies Sedimentarias. Instituto de Geología y Recursos Minerales, Geología Argentina, Anales, 29(16):399-432.

Martinelli, A. 2003. Cranial remains of the bizarre notosuchid Comahuesuchus brachybuccalis (Archosauria, Crocodyliformes) from the Late Cretaceous of Rio Negro Province (Argentina). Ameghiniana, 40(4):559-572.

Ortega, F.; Gasparini, Z.; Buscalioni, A.D. \& Calvo, J.O. 2000.
New species of Araripesuchus (Crocodylomorpha, Mesoeucrocodylia) from the Lower Cretaceous of Patagonia (Argentina). Journal of Vertebrate Paleontology, 20(1):57-76.

Pol, D. 1999a. El esqueleto postcraneano de Notosuchus terrestris (Archosauria: Crocodyliformes) del Cretácico Superior de la Cuenca Neuquina y su información filogenética. Facultad de Ciencias Exactas y Naturales, Universidad de Buenos Aires, Tesis de Licenciatura, 160 p.

Pol, D. 1999b. Basal mesoeucrocodylian relationships: new clues to old conflicts. Journal of Vertebrate Paleontology, 19:69A.

Pol, D. 1999c. Restos postcraneanos de Notosuchus terrestris, características e implicancias en la locomoción. Ameghiniana, 36(1):106.

Pol, D. 2003. New remains of Sphagesaurus hunei (Crocodylomorpha, Mesoeucrocodylia) from the Late Cretaceous of Brazil. Journal of Vertebrate Paleontology, 23(4):817-831. doi: 10.1671/A1015-7

Pol, D. 2005. Postcranial remains of Notosuchus terrestris (Archosauria: Crocodyliformes) form the Upper Cretaceous of Argentina. Ameghiniana, 42(1):21-38.

Pol, D. \& Apesteguia, S. 2005. New Araripesuchus remains from the Early Late Cretaceous (Cenomanian-Turonian) of Patagonia. American Museum Novitates, 3490:1-38. doi: 10.1206/00030082

Pol, D. \& Gasparini, Z. 2007. Crocodyliformes. In: Z. Gasparini; R. Coria \& L. Salgado (eds.) Mesozoic fossil vertebrates from Patagonia, Indiana University Press, p. 116-142.

Pol, D. \& Norell, M. 2004. A new crocodyliform from Zos Canyon, Mongolia. American Museum Novitates, 6445:1-36.

Price, L.I. 1955. Novos crocodilideos dos arenitos de Serie Bauru, Cretáceo do Estado de Minas Gerais. Anais da Academia Brasileira de Ciencias, 27:487-498.

Romer, A.S. 1956. Osteology of the Reptiles. Chicago University Press, Chicago, 772 p.

Sanchez, M.N.; Heredia, S. \& Calvo, J. 2006. Paleoambientes sedimentarios del Cretácico Superior de la Formación Plottier (Grupo Neuquén), Departamento Confluencia, Neuquén. Revista de la Asociación Geológica Argentina, 61(1):3-18.

Sereno, P. C. \& Arcucci, A.B. 1990. The monophyly of crurotarsal archosaurs and the origin of bird and crocodile ankle joints. Neues Jahrbuch für Geologie und Paläeontologie, Abhandlungen, 180:21-52.

Sereno, P.C. \& Larsson, H.C.E. 2009. Cretaceous crocodyliforms from the Sahara. ZooKeys, 28:1-143.

Sereno, P.C.; Sidor, C.A.; Larsson, H.C. E. \& Gado, B. 2003. A new notosuchian from the Early Cretaceous of Niger. Journal of Vertebrate Paleontology, 23(2):477-482. doi: 10.1671/02724634

Turner, A.H. 2004. Crocodyliform biogeography during the Cretaceous: evidence of Gondwana vicariance from biogeographical analysis. Proceedings of the Royal Society $B$, Biological Sciences, 271:2003-2009. doi: 10.1098/rspb.2004.2840

Turner, A.H. 2006. Osteology and phylogeny of a new species of Araripesuchus (Crocodyliformes: Mesoeucrocodylia) from the Late Cretaceous of Madagascar. Historical Biology, 2006:1115. doi: 10.1080/08912960500516112

Turner, A.H. \& Calvo, J. 2005. A new sebecosuchian crocodyliform from the Late Cretaceous of Patagonia. Journal of Vertebrate Paleontology, 25(1):87-98. doi: 10.1671/0272-4634

Woodward A.S. 1896. On two Mesozoic Crocodilians Notosuchus 
(Genus Novum) and Cynodontosuchus (Genus Novum) from the Red Sandstone of the Territory of Neuquen (Argentine Republic). Anales del Museuo de la Plata, Paleontologia Argentina, 6:1-20.

Wu, X.C. \& Sues, H.D. 1996. Anatomy and phylogenetic relationships of Chimaerasuchus paradoxus, an unusual crocodyliform from the Cretaceous of Hubei, China. Journal of Vertebrate Paleontology, 16:688-702.

Received in April, 2010; accepted in November, 2010. 